\title{
Deterministic Nonlinear Transformations of Phase Noise in Quantum-Limited Frequency Combs
}

\author{
A. Liehl, ${ }^{1, \dagger}$ P. Sulzer, ${ }^{1, \dagger}$ D. Fehrenbacher, ${ }^{1}$ T. Rybka, ${ }^{1}$ D. V. Seletskiy, ${ }^{1,2}$ and A. Leitenstorfer ${ }^{1, *}$ \\ ${ }^{1}$ Department of Physics and Center for Applied Photonics, University of Konstanz, D-78457 Konstanz, Germany \\ ${ }^{2}$ Department of Engineering Physics, Polytechnique Montréal, Montréal, H3T 1J4, Canada
}

(Received 31 October 2018; published 24 May 2019)

\begin{abstract}
Optical phase noise of femtosecond lasers is analyzed over various steps of broadband nonlinear frequency conversion. The intrinsic phase jitter of our system originates from quantum statistics in the mode-locked oscillator. Supercontinuum generation by four-wave-mixing processes preserves a noise minimum at the optical carrier frequency. From there, a quadratic increase of the comb linewidth results with mutually anticorrelated phase fluctuations of both spectral wings. Passive phase locking by difference frequency generation strongly enhances the optical phase noise to a level equaling the carrier-envelope phase jitter of the fundamental comb. The same value results from quadratic extrapolation of the optical phase noise to radio frequencies. Our findings are consistent with a fully deterministic transformation of phase noise according to the elastic tape model.
\end{abstract}

DOI: 10.1103/PhysRevLett.122.203902

Mode-locked lasers are powerful tools for high-precision metrology. Their application in time-domain studies of ultrafast phenomena often requires nonlinear frequency conversion to access the resonances of investigated systems. In the extreme limits, attosecond technology provides the fastest electromagnetic transients that can be controlled to date [1]. This area is based on the generation of high optical harmonics to reach the extreme ultraviolet spectral regime. In the opposite direction, few-cycle midinfrared transients emerge from ultrabroadband optical rectification [2], allowing, e.g., active stabilization of quantum cascade lasers [3] and direct detection of the electric field by electro-optic sampling [4,5]. Phase noise of the optical carrier wave ultimately limits the performance of both techniques. This fact is especially relevant for phenomena depending on the subcycle structure of the electromagnetic driving field [6-8].

The advent of femtosecond frequency combs has extended the relevance of mode-locked lasers far into spectral-domain precision metrology $[9,10]$. Combining them with microwave technologies yields the best time standards currently available. Extremely narrow-band atomic transitions represent a key element of these optical atomic clocks $[11,12]$. Such high-end applications require absolute stabilization of all comb modes while maintaining optimum noise performance. This goal necessitates sensitive control over 2 degrees of freedom, namely, the pulse repetition rate of the oscillator $f_{\text {rep }}$ and the carrier-envelope offset (CEO) frequency $f_{\mathrm{CEO}}$. Currently, fractional uncertainties of $f_{\text {CEO }}$ below $10^{-20}$ [13] as well as narrow optical comb modes with only a few millihertz bandwidth are supported by both actively controlled solid-state [14] and fiber laser systems [15]. In particular, most ultrastable and precise sources are based on mode-locked Ti:sapphire and Er:fiber oscillators with broadband gain spectra centered at $370 \mathrm{THz}$ (wavelength of $810 \mathrm{~nm}$ ) and $193 \mathrm{THz}(1550 \mathrm{~nm})$, respectively. Again, nonlinear frequency conversion is central to reach atomic transitions in the ultraviolet and visible range [16]. Even detection and stabilization of $f_{\text {CEO }}$ itself relies heavily on spectral broadening via self-phase modulation and second-harmonic generation $[9,10,17,18]$. Optical nonlinearities also allow passive elimination of $f_{\text {CEO }}$ [19] instead of its active stabilization [20]. This step results in a train of ultrashort pulses with identical electric field trace and ultralow shot-to-shot carrier-envelope phase jitter [21].

The influence of nonlinear frequency conversion processes is of paramount importance not only for expanding the limits in frequency metrology $[16,22]$ but also in a wide range of advanced applications like, e.g., ultrabroadband spectroscopy [23] or studies on fundamental physics [24]. Phase noise is particularly crucial in both linear [25] and nonlinear [26,27] cavity-enhanced comb spectroscopy since it is converted to amplitude fluctuations, thus reducing the signal-to-noise ratio. All these examples motivate a detailed analysis of the spectral distribution of the phase noise in mode-locked lasers following the required steps of nonlinear frequency conversion.

In this Letter, we examine the evolution of phase noise and its correlations in the limit where the performance of mode-locked lasers is dominated by elementary quantum processes. The setup of ultrafast laser systems used in our experiments is sketched in Fig. 1(a) [7,28]. A solitonic Er: fiber oscillator emits a train of Fourier-limited ultrashort pulses whose spectra exhibit a full width at half maximum 


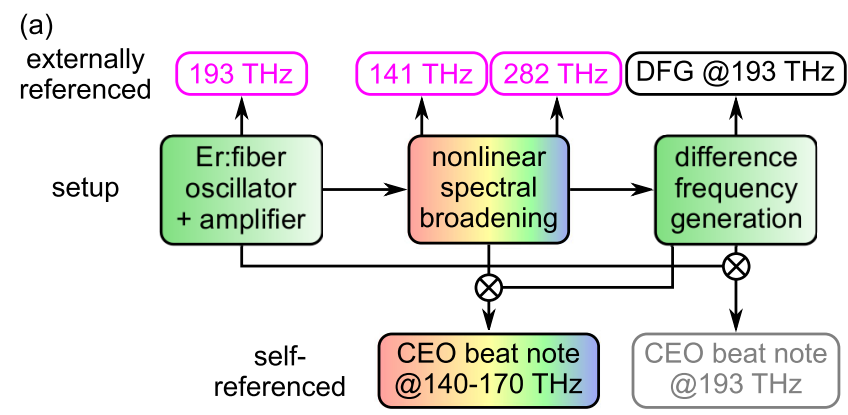

(b) externally
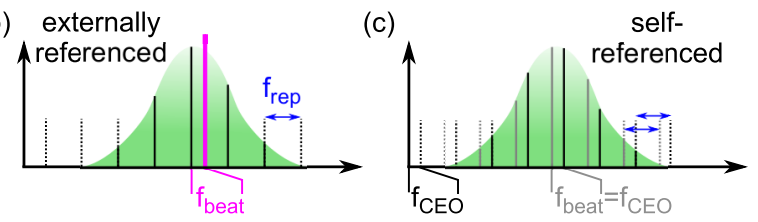

FIG. 1. (a) Scheme of the mode-locked Er:fiber laser system and setup for broadband investigation of phase noise and linewidth characteristics. Single-line references and their subharmonics are depicted in magenta. (b) Spectral scheme of generating a radio-frequency beat $f_{\text {beat }}$ between a narrow-line reference and the closest line in a frequency comb with a spacing given by the laser repetition rate $f_{\text {rep }}$. (c) Spectral scheme when temporally interfering the fundamental comb where $f_{\text {CEO }} \neq 0$ with the phase-stable comb generated via DFG $\left(f_{\mathrm{CEO}}=0\right)$, thus providing access to the carrier-envelope offset frequency $f_{\mathrm{CEO}}$.

(FWHM) of up to $18 \mathrm{~nm}$. Subsequently, an Er:fiber amplifier boosts pulse energies close to $10 \mathrm{~nJ}$ and a coherent supercontinuum with frequency content ranging from 140 to $370 \mathrm{THz}$ is generated in a highly nonlinear germanosilicate bulk fiber [29]. Passive elimination of $f_{\text {CEO }}$ is accomplished by difference frequency generation (DFG) between a dispersive wave centered at a frequency of $\nu_{\text {disp }}=350 \mathrm{THz}$ and a soliton around $\nu_{\text {sol }}=157 \mathrm{THz}$. The mixing product exhibits a carrier frequency of $193 \mathrm{THz}$, corresponding to a center wavelength of $1.55 \mu \mathrm{m}$, thus enabling efficient reamplification in Er:fiber [19].

In order to analyze the phase noise $\Delta \phi(t)$ and linewidth $\delta$ of single comb modes, we generate beat notes at different outputs of our laser system. This technique converts the desired information from the optical to the radio frequency range, which is accessible electronically. Specifically, we superimpose low-noise external continuous-wave laser references [magenta boxes in Fig. 1(a)] with the nearest comb line [see spectral scheme in Fig. 1(b)]. Alternatively, the offset-free and the fundamental comb may be brought to interference [Fig. 1(c)]. In this case, the beat frequency $f_{\text {beat }}$ is centered at $f_{\mathrm{CEO}}$.

The time-domain phase noise $\Delta \phi(t)$ of each beat note is now extracted by recording a time series in the radiofrequency range and applying a modified Takeda algorithm [30]. The optical phase noise spectral density $S_{\Delta \phi}(f)$ at a given measurement bandwidth $B$ is then defined by [31]

$$
S_{\Delta \phi}(f)=|F T[\Delta \phi(t)]|^{2} B^{-1} .
$$

The spectral envelope of the corresponding comb line may be derived from $S_{\Delta \phi}(f)$. Generally, this step depends on the measurement observation time set to $12 \mathrm{~ms}$ in all experimental data presented here [32]. For quantum-limited performance, the frequency noise spectral density $S_{\Delta \nu}(f)$ remains constant [33] and

$$
S_{\Delta \nu}(f)=f^{2} S_{\Delta \phi}(f)=S_{0} .
$$

In this case, the line shape converges to a Lorentzian with a FWHM of [32]

$$
\delta=\pi S_{0}
$$

in the limit of infinitely long observation time.

Applying this methodology, we now compare two offset-free frequency comb systems with identical general design but operating at different repetition rates of 80 and $100 \mathrm{MHz}$, respectively. The optical linewidth of both DFG combs $\left(\delta_{\mathrm{DFG}}\right)$ is determined at a frequency of $193 \mathrm{THz}$ [see Fig. 1(a) top right and Fig. 1(b)]. Figure 2 shows $\delta_{\mathrm{DFG}}$ of both sources as a function of optical bandwidth $\Delta \nu$ of the fundamental soliton circulating in the oscillators which we control via the optical pump power [28]. For a quantumlimited phase jitter, $\delta_{\mathrm{DFG}}$ is expected to be proportional to the square of the pulse duration of the Fourier-limited intracavity soliton [34,35]: $\delta_{\mathrm{DFG}} \propto(\Delta \nu)^{-2}$. Both DFG combs show excellent agreement to the same numerical fit corresponding to this prediction (black dashed in Fig. 2). Potential sources of quantum noise which can cause such behavior include, e.g., amplified spontaneous emission or optical shot noise. Specifically, intensity variations originating from the quantum fluctuations of the intracavity photon number convert into a timing jitter via the nonlinear refractive index change in the cavity elements. Such

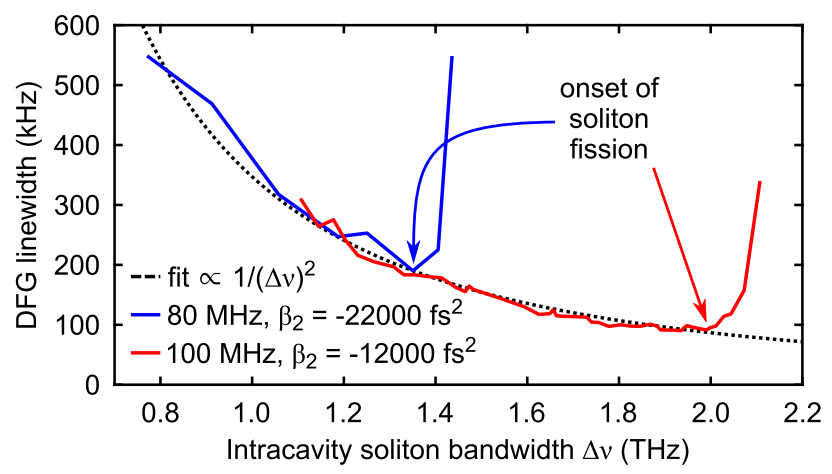

FIG. 2. Evolution of the optical linewidth of two DFG combs (blue and red) at $193 \mathrm{THz}$ when the spectral width $\Delta \nu$ of the soliton within the all-fiber oscillator is varied. Both fit well to the theoretical prediction for quantum-limited phase noise characteristics (black dashed) up to the point where intracavity soliton fission crucially affects the pulse characteristics. 
shot-noise-induced phase fluctuations are often referred to as Gordon-Haus jitter [36]. They dominate in mode-locked fiber sources due to the moderate intracavity pulse energies and large nonlinear interaction length in the dielectric waveguides. Only if soliton fission emerges, a steep increase of $\delta_{\mathrm{DFG}}$ arises due to abrupt changes of the pulse properties. Since the net second-order dispersion $\beta_{2}$ is lower for the $100 \mathrm{MHz}$ system (see Fig. 2), soliton fission occurs at a higher bandwidth $\Delta \nu$.

Although the minimum DFG linewidth of the $100 \mathrm{MHz}$ offset-free comb is below $100 \mathrm{kHz}$, we expect the fundamental comb to show significantly less optical phase noise since even non-quantum-limited sources have been reported to exhibit considerably lower phase jitter [37]. Indeed, a broadband study of the noise characteristics of comb lines at 141, 193, and $282 \mathrm{THz}$ presented recently [28] confirms this assumption. The linewidths of the corresponding comb modes are depicted in magenta in Fig. 3(a) as a function of optical frequency. The leastsquare fit (cyan solid line) demonstrates a quadratic increase of the comb linewidth away from the optical carrier at $193 \mathrm{THz}$, as it is predicted for quantum-limited timing jitter [33]. Qualitatively, such behavior can be explained by an elastic tape picture which describes absolute frequency fluctuations of the comb teeth as a breathing motion with a specific fix point $n_{\text {fix }}$, that is, the number of the longitudinal comb mode with lowest phase noise [38-40]. These correlations are a fundamental consequence of the fact that all longitudinal comb modes share the same lasing cavity. In the case of a quantum-limited phase jitter, $n_{\text {fix }}$ coincides with the carrier $n_{c}$ as illustrated in Fig. 3(c), which agrees with our results presented in Fig. 3(a).
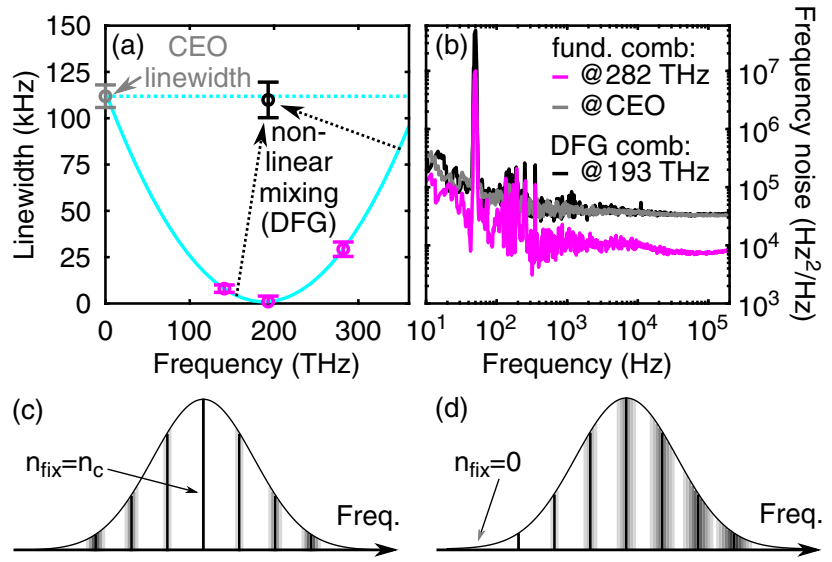

FIG. 3. (a) Spectral width of several individual comb teeth. $f_{\text {CEO }}$ (gray) is measured by interfering the fundamental and DFG comb. The linewidth of the latter at $193 \mathrm{THz}$ is shown in black. (b) Frequency noise spectral density of a comb line at $282 \mathrm{THz}$ (pink), $f_{\mathrm{CEO}}$ (gray) and the DFG comb at $193 \mathrm{THz}$ (black). (c) and (d) Sketch of the elastic tape behavior of the comb modes for a fix point at the carrier and zero frequency, respectively.
A more detailed analysis of the evolution of the comb linewidth during spectral conversion is provided by studying the frequency noise spectral density $S_{\Delta \nu}(f)$ of individual modes. The pink graph in Fig. 3(b) represents $S_{\Delta \nu}(f)$ of a mode of the fundamental comb at $282 \mathrm{THz}$. Its broadband flat shape further supports the quantum origin of the intrinsic optical phase noise of the modelocked Er:fiber oscillator [33]. Interestingly, the noise spectrum of the DFG comb at $193 \mathrm{THz}$ remains white but its level is significantly increased [28]. Therefore, nonlinear processes seem to crucially affect phase noise but in a way that it is still governed by its quantum origin. The linewidth of the corresponding tooth of the DFG comb is determined to be $110 \pm 10 \mathrm{kHz}$ [black marker in Fig. 3(a)]. It is worth to emphasize that this value is significantly higher than the sum of the linewidths of the two DFG inputs at 157 and $350 \mathrm{THz}$ extracted from the quadratic fit (black dotted arrows). This finding cannot be attributed to potentially imperfect cancellation of $f_{\mathrm{CEO}}$ which is negligible [21]. Instead, it must originate from strong correlations between all longitudinal modes. In the following, we quantitatively discuss the underlying physics using the elastic tape picture [38].

First, the phase noise spectral density $S_{\Delta \phi, n}$ of individual modes $n$ of our fundamental Er:fiber comb can be expressed as [37]

$$
S_{\Delta \phi, n}=S_{\Delta \phi, \text { rep }}\left(n-n_{c}\right)^{2}+S_{\Delta \phi, c} .
$$

Here, $S_{\Delta \phi, \text { rep }}(f)$ and $S_{\Delta \phi, c}(f)$ are the fundamental fluctuations of the repetition rate and $f_{\mathrm{CEO}}$, respectively. Because of the quantum origin of the phase noise of our mode-locked Er:fiber source, Eqs. (2) and (3) apply. Therefore, the same relation holds for the linewidth of single comb teeth [41]:

$$
\delta_{n}=\delta_{\text {rep }}\left(n-n_{c}\right)^{2}+\delta_{c} .
$$

Here, $\delta_{\text {rep }}$ and $\delta_{c}$ are defined analogously. This result precisely explains the quadratic behavior found in Fig. 3(a). It quantitatively describes the broadening of the comb modes during supercontinuum generation by four-wavemixing processes.

Considering three-wave-mixing, the time domain phase fluctuations of the involved driving fields add linearly. Particularly, for DFG between the $n$th and $m$ th comb line, we have [42]

$$
\Delta \phi_{\mathrm{DFG}}(t)=\Delta \phi_{n}(t)-\Delta \phi_{m}(t) .
$$

Following Eq. (1), a cross-correlation between the two input waves results:

$$
\begin{aligned}
S_{\Delta \phi, \mathrm{DFG}} & =\left|F T\left[\Delta \phi_{n}(t)-\Delta \phi_{m}(t)\right]\right|^{2} B^{-1} \\
& =S_{\Delta \phi, n}+S_{\Delta \phi, m}-2 \chi_{\mathrm{DFG}} \sqrt{S_{\Delta \phi, n} S_{\Delta \phi, m}} .
\end{aligned}
$$


The correlation parameter $\chi_{\mathrm{DFG}}$ vanishes for mutually incoherent inputs. Within the spectrum of a frequency comb, instead, $\chi_{\mathrm{DFG}}$ is determined by means of the elastic tape picture [38]. Accordingly, we expect fully correlated phase fluctuations $\left(\chi_{\mathrm{DFG}}=1\right)$ if the frequencies of both involved comb modes reside below or above the fix point. In our DFG implementation, the driving fields, i.e., dispersive wave and the soliton from the broadband supercontinuum, satisfy $n_{\text {disp }}>n_{c}>n_{\text {sol }}$, corresponding to an anticorrelated behavior with $\chi_{\mathrm{DFG}}=-1$. By combining Eqs. (3) and (7), the calculation of the DFG linewidth yields [43]

$$
\begin{aligned}
\delta_{\mathrm{DFG}} & =\delta_{\mathrm{disp}}+\delta_{\mathrm{sol}}-2 \chi_{\mathrm{DFG}} \sqrt{\delta_{\mathrm{disp}} \delta_{\mathrm{sol}}} \\
& =\delta_{\mathrm{rep}}\left(n_{\mathrm{disp}}-n_{\mathrm{sol}}\right)^{2} .
\end{aligned}
$$

Here, $\delta_{\text {disp }}$ and $\delta_{\text {sol }}$ are given by Eq. (5) while we have neglected $\delta_{c}$ which is a suitable approximation due to the ultralow phase noise of our Er:fiber oscillator as compared to the DFG linewidth [see Fig. 3(a)]. Consequently, the DFG comb exhibits a fix point at zero frequency [44] where the linewidth is negligible [21]. This behavior contrasts the spectral dynamics of the fundamental comb [see Figs. 3(a) and 3(c)]. It is depicted schematically in Fig. 3(d). Given that $n_{\text {disp }}-n_{\text {sol }}=n_{c}$ for our offset-free Er:fiber frequency comb, we conclude that its optical linewidth matches $\delta_{\mathrm{DFG}}=\delta_{\text {rep }} n_{c}^{2}$. This is precisely the zero crossing $\delta_{0}$ of the quadratic increase of the linewidth of the fundamental comb as described theoretically in Eq. (5). In Fig. 3(a), the level of $\delta_{0}$ extracted from the quadratic fit (cyan solid graph) to our measurements is highlighted as a cyan dotted line. We find excellent agreement between $\delta_{0}$ and the $\delta_{\mathrm{DFG}}$ determined experimentally [black marker in Fig. 3(a)]. This result underlines that our theoretical modeling describes the evolution of phase noise in four-wave-mixing processes and DFG. Its extension to arbitrary nonlinear optical processes is straightforward.

Since the first mode of the fundamental comb is located at $f_{\mathrm{CEO}}$, the zero crossing $\delta_{0}$ of the comb linewidth might be accessed experimentally by a beat note centered at this frequency [37,45], denoted as CEO beat note in the following. In our implementation, we check this assumption by superimposing the fundamental and DFG comb [see spectral scheme in Fig. 1(c)]. Indeed, this measurement reveals a CEO linewidth of $112 \pm 6 \mathrm{kHz}$ [gray marker in Fig. 3(a)], which is identical with $\delta_{\text {DFG }}$ and $\delta_{0}$. Likewise, the frequency noise spectral density of the CEO beat note [gray in Fig. 3(b)] strongly matches the one of the DFG comb at $193 \mathrm{THz}$ (black). However, due to the correlations between all comb modes arising during nonlinear frequency conversion discussed above, it is not trivial that the linewidth of the CEO beat note is independent of the spectral parts exploited for its generation. We test this question experimentally by detuning the central frequency of the DFG comb away from $193 \mathrm{THz}$. To this end, the spectra of the involved driving fields are narrowed to only a few $\mathrm{THz}$ bandwidth as shown in Figs. 4(a) and 4(b) while the phase-matching condition for DFG is adjusted properly. For each pair depicted in the same color, the dispersive wave [Fig. 4(a)] is centered at double frequency of the soliton of the supercontinuum [Fig. 4(b)]. Hence, the DFG target frequency is given by

$$
\nu_{\mathrm{DFG}}=\nu_{\mathrm{disp}}-\nu_{\mathrm{sol}}=2 \nu_{\mathrm{sol}}-\nu_{\mathrm{sol}}=\nu_{\mathrm{sol}} .
$$

Therefore, various CEO beat notes can be generated by spatiotemporally overlapping the DFG output and the soliton at a photo detector. As demonstrated in Fig. 4(c), the width of all CEO beat notes is independent of the central frequency of the DFG when varied between 140 and $170 \mathrm{THz}$. Additionally, all these results coincide with (i) the value obtained by superposition of the DFG comb if centered at $193 \mathrm{THz}$ and the mode-locked oscillator [gray marker in Fig. 4(c)] and (ii) the measurement uncertainty range of $\delta_{\mathrm{DFG}}$ at a frequency of $193 \mathrm{THz}$ (black lines and shading). The overlap of the frequency noise spectra of $f_{\text {CEO }}$ shown in Fig. 4(d) for two different DFG target frequencies agrees with this finding.

For a full theoretical description and generalization of this observation, we again consider the elastic tape

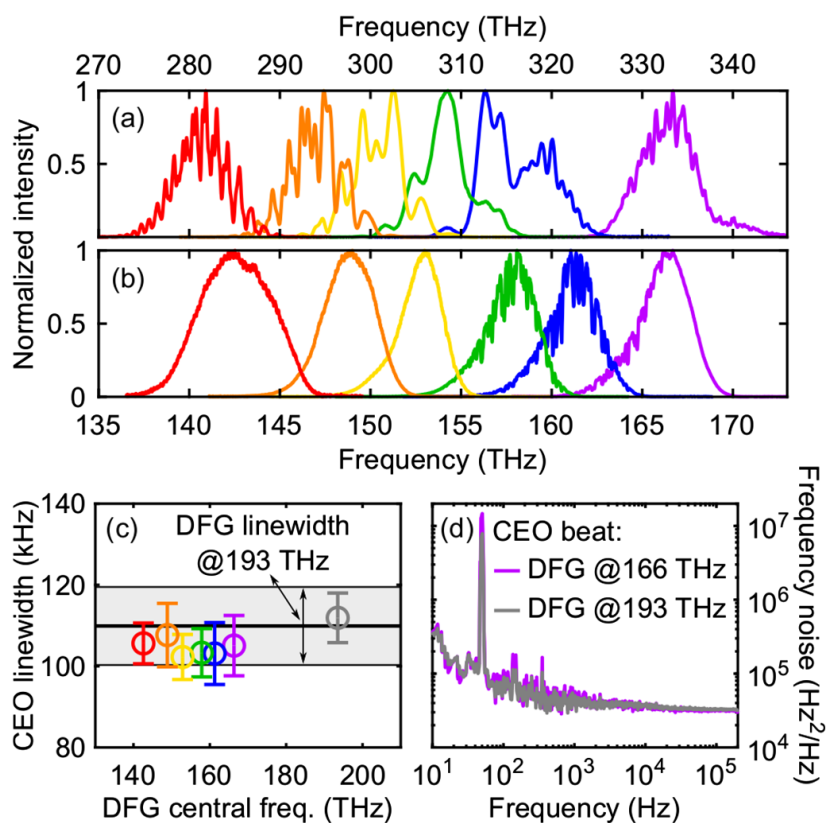

FIG. 4. (a) and (b) Narrowed spectra of the dispersive wave and the soliton, respectively, used for DFG at varying frequencies. (c) CEO linewidth when the DFG is centered at $193 \mathrm{THz}$ (gray marker) and if mixed with spectra of same color shown in (a) and (b). Black line and shading depict the optical linewidth of the DFG comb at $193 \mathrm{THz}$ and its statistical uncertainty, respectively. (d) Frequency noise spectral density of $f_{\mathrm{CEO}}$ if the DFG is centered at two different spectral regions. 
model [38]. Since the CEO beat note is obtained by heterodyne mixing of the soliton from the supercontinuum with the DFG output, their phase noise adds similarly to Eq. (6):

$$
\Delta \phi_{\mathrm{CEO}}(t)=\Delta \phi_{\mathrm{DFG}}(t)-\Delta \phi_{\mathrm{sol}}(t)
$$

By analogy with Eqs. (7) and (8), the linewidth of the corresponding CEO beat note is given by

$$
\delta_{\mathrm{CEO}}=\delta_{\mathrm{DFG}}+\delta_{\mathrm{sol}}-2 \chi_{\mathrm{het}} \sqrt{\delta_{\mathrm{DFG}} \delta_{\mathrm{sol}}},
$$

where $\chi_{\text {het }}$ is the correlation between the DFG comb and the soliton of the fundamental comb occurring during heterodyne mixing. Assuming $\chi_{\text {het }}= \pm 1$, we obtain $\delta_{\mathrm{CEO}}=$ $\delta_{\text {rep }} n_{c}^{2}=\delta_{0}$ [43]. Although both $\delta_{\text {sol }}$ and $\delta_{\mathrm{DFG}}$ strongly depend on the optical frequency [Eqs. (5) and (8)], our consideration predicts $\delta_{\mathrm{CEO}}$ to be independent of the spectral components involved in the nonlinear processes to generate the CEO beat note. This finding fully explains our results shown in Figs. 4(c) and 4(d). Note that the identity $\delta_{\mathrm{CEO}}=\delta_{\mathrm{rep}} n_{c}^{2}$ can also be derived with our model if $\delta_{\text {CEO }}$ is determined with an $f$-to- $2 f$ interferometer or other schemes, e.g., $3 f$-to- $2 f$ configurations. We further stress that our calculations are even valid for non-quantumlimited sources if the phase noise spectral density instead of the bare CEO linewidth is considered, thus $S_{\Delta \phi \text {,epp }} n_{c}^{2}=$ $S_{\Delta \phi, 0}=S_{\Delta \phi, \mathrm{CEO}}$. Since the width of the comb line at $f_{\mathrm{CEO}}$ can be derived from this quantity [32], our results demonstrate that the linewidth of a CEO beat note is truly independent of the scheme applied for its generation as long as the detection method fully maintains coherence. Therefore, $\delta_{\mathrm{CEO}}$ can generally be obtained by extrapolating $\delta_{n}$ [see Eq. (5)] to zero frequency.

In summary, we have investigated the coherent transfer of phase noise during nonlinear frequency conversion, resulting in strong correlations across the spectrum of a mode-locked laser. Quantitative modeling based on the elastic tape behavior of all comb modes describes multiple-wave-mixing processes between arbitrary spectral components. We have demonstrated unrestricted validity along the examples of DFG, four-wave interactions and the measurement of $f_{\mathrm{CEO}}$ using home-built femtosecond Er:fiber lasers. The noise performance of these systems is limited by quantum processes. Nevertheless, our considerations may be easily extended to other noise components as well as to nonlinear optical processes of arbitrary order, thus describing any situation where the stability of a frequency comb is relevant.

Funding by the ERC (Advanced Grant No. 290876 "UltraPhase") is gratefully acknowledged.

*alfred.leitenstorfer@uni-konstanz.de

${ }^{\dagger}$ These authors contributed equally to this work.
[1] F. Krausz and M. Ivanov, Rev. Mod. Phys. 81, 163 (2009).

[2] A. Bonvalet, M. Joffre, J. L. Martin, and A. Mignus, Appl. Phys. Lett. 67, 2907 (1995).

[3] L. Consolino et al., Nat. Commun. 3, 1040 (2012).

[4] Q. Wu and X.-C. Zhang, Appl. Phys. Lett. 67, 3523 (1995).

[5] R. Huber, A. Brodschelm, F. Tauser, and A. Leitenstorfer, Appl. Phys. Lett. 76, 3191 (2000).

[6] M. Krüger, M. Schenk, and P. Hommelhoff, Nature (London) 475, 78 (2011).

[7] T. Rybka, M. Ludwig, M. F. Schmalz, V. Knittel, D. Brida, and A. Leitenstorfer, Nat. Photonics 10, 667 (2016).

[8] C. Riek, P. Sulzer, M. Seeger, A. S. Moskalenko, G. Burkard, D. V. Seletskiy, and A. Leitenstorfer, Nature (London) 541, 376 (2017).

[9] D. J. Jones, S. A. Diddams, J. K. Ranka, A. Stentz, R. S. Windeler, J. L. Hall, and S. T. Cundiff, Science 288, 635 (2000).

[10] R. Holzwarth, Th. Udem, T. W. Hänsch, J. C. Knight, W. J. Wadsworth, and P. St. J. Russell, Phys. Rev. Lett. 85, 2264 (2000).

[11] S. A. Diddams et al., Science 293, 825 (2001).

[12] M. Takamoto, F.-L. Hong, R. Higashi, and H. Katori, Nature (London) 435, 321 (2005).

[13] H. Leopardi, J. Davila-Rodriguez, F. Quinlan, J. Olson, J. A. Sherman, S. A. Diddams, and T. M. Fortier, Optica 4, 879 (2017).

[14] S. T. Cundiff and J. Ye, Rev. Mod. Phys. 75, 325 (2003).

[15] J. Kim and Y. Song, Adv. Opt. Photonics 8, 465 (2016).

[16] A. D. Ludlow, M. M. Boyd, J. Ye, E. Peik, and P. O. Schmidt, Rev. Mod. Phys. 87, 637 (2015).

[17] F. Tauser, A. Leitenstorfer, and W. Zinth, Opt. Express 11, 594 (2003).

[18] B. R. Washburn, S. A. Diddams, N. R. Newbury, J. W. Nicholson, M. F. Yan, and C. G. Jorgensen, Opt. Lett. 29, 250 (2004).

[19] G. Krauss, D. Fehrenbacher, D. Brida, C. Riek, A. Sell, R. Huber, and A. Leitenstorfer, Opt. Lett. 36, 540 (2011).

[20] S. Koke, C. Grebing, H. Frei, A. Anderson, A. Assion, and G. Steinmeyer, Nat. Photonics 4, 462 (2010).

[21] A. Liehl, D. Fehrenbacher, P. Sulzer, A. Leitenstorfer, and D. V. Seletskiy, Opt. Lett. 42, 2050 (2017).

[22] Th. Udem, R. Holzwarth, and T. W. Hänsch, Nature (London) 416, 233 (2002).

[23] I. Coddington, N. Newbury, and W. Swann, Optica 3, 414 (2016).

[24] R. M. Godun, P. B. R. Nisbet-Jones, J. M. Jones, S. A. King, L. A. M. Johnson, H. S. Margolis, K. Szymaniec, S. N. Lea, K. Bongs, and P. Gill, Phys. Rev. Lett. 113, 210801 (2014).

[25] F. Adler, M. J. Thorpe, K. C. Cossel, and J. Ye, Ann. Rev. Anal. Chem. 3, 175 (2010).

[26] M. A. R. Reber, Y. Chen, and T. K. Allison, Optica 3, 311 (2016).

[27] T. K. Allison, J. Phys. B 50, 044004 (2017).

[28] D. Fehrenbacher, P. Sulzer, A. Liehl, T. Kälberer, C. Riek, D. V. Seletskiy, and A. Leitenstorfer, Optica 2, 917 (2015).

[29] D. Brida, G. Krauss, A. Sell, and A. Leitenstorfer, Laser Photonics Rev. 8, 409 (2014).

[30] M. Takeda, H. Ina, and S. Kobayashi, J. Opt. Soc. Am. 72, 156 (1982). 
[31] IEEE Standards Coordinating Committee 27, in 1139-2008 IEEE Standard Definitions of Physical Quantities for Fundamental Frequency and Time Metrology-Random Instabilities (IEEE, New York, 2009), pp. c1-35, https:// ieexplore.ieee.org/document/4797525.

[32] D. S. Elliott, R. Roy, and S. J. Smith, Phys. Rev. A 26, 12 (1982).

[33] N. R. Newbury and W. C. Swann, J. Opt. Soc. Am. B 24, 1756 (2007).

[34] H. A. Haus and A. Mecozzi, IEEE J. Quantum Electron. 29, 983 (1993).

[35] R. Paschotta, Appl. Phys. B 79, 163 (2004).

[36] J. P. Gordon and H. A. Haus, Opt. Lett. 11, 665 (1986).

[37] J. J. McFerran, W. C. Swann, B. R. Washburn, and N. R. Newbury, Opt. Lett. 31, 1997 (2006).

[38] H. R. Telle, B. Lipphardt, and J. Stenger, Appl. Phys. B 74, 1 (2002).
[39] E. Benkler, H. R. Telle, A. Zach, and F. Tauser, Opt. Express 13, 5662 (2005).

[40] F. Cappelli et al., Laser Photonics Rev. 10, 623 (2016).

[41] F. X. Kärtner, U. Morgner, T. Schibli, R. Ell, H. A. Haus, J. G. Fujimoto, and E. P. Ippen, in Few-Cycle Laser Pulse Generation and Its Applications, edited by F. X. Kärtner (Springer, Berlin, Heidelberg, 2004).

[42] G. Cerullo, A. Baltuska, O. D. Mücke, and C. Vozzi, Laser Photonics Rev. 5, 323 (2011).

[43] See Supplemental Material at http://link.aps.org/ supplemental/10.1103/PhysRevLett.122.203902 for a full derivation of these relations.

[44] T. Puppe, A. Sell, R. Kliese, N. Hoghooghi, A. Zach, and W. Kaenders, Opt. Lett. 41, 1877 (2016).

[45] R. Paschotta, A. Schlatter, S. C. Zeller, H. R. Telle, and U. Keller, Appl. Phys. B 82, 265 (2006). 